\title{
Etat nutritionnel des enfants de moins de 5 ans reçus à l'Unité de Vaccination du Centre de Santé de Hounsouko à Porto-Novo (Sud du Bénin)
}

\author{
Abdou Ganiou YESSOUFOU ${ }^{1 *}$, Abèbi Karimath YESSOUFOU ${ }^{2}$, \\ Adégnika Amirath ADEBO ${ }^{1}$, Laure EGOUNLETY ${ }^{3}$ et Alphonse SEZAN ${ }^{1}$ \\ ${ }^{1}$ Laboratoire de Biomembranes et de Signalisation Cellulaire, Département de Physiologie Animale, Faculté \\ des Sciences et Techniques, Université d'Abomey-Calavi, BP 526 Cotonou, République du Bénin. \\ ${ }^{2}$ Clinique de Pédiatrie et Néonatologie de Sènadé 06 BP 601 Cotonou, République du Bénin. \\ ${ }^{3}$ Centre de Santé de Hounsouko, Zone Sanitaire Porto-Novo/Aguégués/Sèmè-Podji, Porto-Novo, République du \\ Bénin. \\ *Auteur correspondant, E-mail : yessoufouga@yahoo.fr/yessoufou601@gmail.com; \\ Tél : (00229) 97441045/(00229) 61788161
}

\section{RESUME}

La malnutrition constitue un problème de santé publique dans les pays en développement. La fréquentation des enfants dans les services de vaccination est une opportunité pour apprécier leur état nutritionnel. Il était question dans cette étude, d'évaluer l'état nutritionnel des enfants de moins de 5 ans reçus à l'Unité de Vaccination du Centre de Santé de Hounsouko à Porto-Novo. Il s'agit d'une étude transversale à visée descriptive et analytique portée sur 350 enfants de 0 à 59 mois reçus dans ledit centre. Les données anthropométriques ont été collectées. A partir de ces données, ont été calculés les indices Poids-pour-Taille, Taille-pour-Age, Poids-pour-Age. Ces données ont été traitées et analysées par les logiciels WHO Anthro®. Il ressort des résultats que 7,4\% des enfants étaient émaciés, 30,3\% présentaient un retard de croissance et 12,3\% avaient une insuffisance pondérale. Par contre, $45,9 \%$ des enfants présentaient une surcharge pondérale dont $11,7 \%$ d'obèses. Par ailleurs, la surcharge pondérale touchait $65 \%$ des enfants de moins 5 mois. Les courbes de distribution des enfants comparativement aux courbes de référence sont décalées vers la gauche pour P/A et $\mathrm{T} / \mathrm{A}$, et vers la droite pour $\mathrm{P} / \mathrm{T}$ évoquant respectivement une tendance à un état de sous-nutrition et de surnutrition.

(C) 2016 International Formulae Group. All rights reserved.

Mots clés: Bénin, état nutritionnel, émaciation, retard de croissance, insuffisance pondérale, double fardeau "malnutrition".

\section{Nutritional status of children under 5 years received Immunization Unit of the Health Center of Hounsouko in Porto-Novo (Benin Southern)}




\section{ABSTRACT}

Malnutrition is a public health problem in developing countries. The attendance of children in immunization services is an opportunity to appreciate their nutritional status. At issue in this study to assess the nutritional status of children under 5 years received Immunization Unit of the Health Center of Hounsouko in Porto-Novo. This is a cross-sectional study descriptive and analytical scope covered 350 children aged 0 to 59 months received in said center. Anthropometric data were collected. From these data were calculated indices Weight for Height, Height-for-age, weight-for-age. These data were processed and analyzed by the WHO Anthro ${ }^{\circledR}$ software. The results show that $7.4 \%$ of children were emaciated, $30.3 \%$ were stunted and $12.3 \%$ were underweight. By cons, $45.9 \%$ of children were overweight with $11.7 \%$ obese. Furthermore, overweight touched $65 \%$ of children aged 5 months less. Distribution of children compared to reference curves are shifted to the left to P/A and T/A, and right for P/T respectively evoking a tendency to a state of under nutrition and over-nutrition.

(C) 2016 International Formulae Group. All rights reserved.

Keywords: Benin, nutritional status, wasting, stunting, underweight, double burden "malnutrition".

\section{INTRODUCTION}

L'état nutritionnel peut être défini comme le statut physique d'un individu, déterminé par la relation entre l'apport et les besoins en nutriments, et par la capacité de l'organisme à digérer, à absorber et à utiliser ces nutriments (Perlemuter, 2002). Selon les estimations de l'UNICEF, 185 millions d'enfants de moins de cinq (05) ans souffrent d'un retard de croissance et 129 millions d'insuffisance pondérale dans les pays en développement (Rice et al., 2004).

En Afrique, la prévalence de retard de croissance est de $40 \%$ et celle de l'insuffisance pondérale de $25 \%$ (UNICEF, 2009).

Au Bénin, 37\% des enfants de 6 à 59 mois accusent un retard de croissance dont $12 \%$ sous forme sévère. Les prévalences de l'insuffisance pondérale et de l'émaciation sont respectivement de $17,3 \%$ et $4,7 \%$ (AGVSA, 2014).

Ce niveau de malnutrition chronique est considéré comme élevé et est révélateur d'une situation d'insécurité alimentaire chronique. En effet, Au Bénin, 23\% des ménages ont une consommation alimentaire inadéquate ne leur permettant pas de mener une vie saine et active.

Dans l'0uémé, la prévalence de retard de croissance est de 30\% chez les enfants de 6 à 59 mois. Ce taux de malnutrition chronique constitue une situation nutritionnelle grave selon les seuils établis par l'OMS. Par contre celle de l'émaciation est de $6,1 \%$ ce qui met l'Ouémé comme le $3^{\text {ème }}$ Département le plus touché par l'émaciation après l'Atacora $(7,8 \%)$ et le Plateau $(6,4 \%)$ et représente ainsi une zone à risque (AGVSA, 2014).

Dans le souci d'avoir une idée sur la situation nutritionnelle des enfants, nous nous sommes proposés d'évaluer leur état nutritionnel dans une structure sanitaire précisément ceux reçus pour la vaccination. En matière de contact avec le système de santé, la fréquentation des enfants de moins de cinq ans dans les unités de vaccination est une opportunité pour apprécier leur état nutritionnel et susciter des recommandations appropriées à l'endroit de leurs parents. Malheureusement peu d'études ont été réalisées pour saisir cette opportunité. Par ailleurs, Les normes précédemment utilisées pour l'évaluation de l'état nutritionnel des enfants sont basées sur la croissance des enfants nourris au lait maternisé (lait de substitution) alors que les nouvelles normes de croissance et de développement de l'enfant recommandées actuellement par l'OMS (OMS, 2010; Sakineh et al., 2014) sont basées sur l'allaitement maternel comme norme biologique. Ces nouvelles normes permettent de plaider en faveur de la protection et de la promotion de l'allaitement 
maternel et d'une alimentation complémentaire adéquate (OMS, 2011).

\section{MATERIEL ET METHODES}

L'enquête s'est déroulée de janvier à avril 2016 à l'Unité de Vaccination du Centre de Santé de Hounsouko à Porto-Novo, Cheflieu du Département de l'Ouémé. Il s'agit d'une étude transversale à visée descriptive et analytique par voie de questionnaire et des mesures anthropométriques sur 350 enfants de 0 à 59 mois révolus considérés comme sains par leurs parents, reçus pour la vaccination dans ledit centre pendant la période d'étude. N'ont pas été inclus dans l'étude les enfants présentant des pathologies diverses.

\section{Taille de l'échantillon}

La taille minimale de l'échantillon a été déterminée selon la formule $\mathrm{N}=\mathrm{t}^{2} \times \mathrm{p}(1-\mathrm{p}) / \mathrm{d}^{2}$ où $\mathrm{N}=$ taille minimum recherchée pour l'échantillon; $\mathrm{t}=$ niveau de confiance à $95 \%$ (valeur type de 1,96); p = prévalence estimative de la malnutrition dans la zone d'étude ; d = marge d'erreur à 5\% (valeur de 0,05). Selon l'Analyse Globale de la Vulnérabilité, de la Sécurité alimentaire et de la Nutrition (AGVSA, 2014), la population d'enfants de moins de 5 ans présentant une malnutrition chronique dans le Département de l'Ouémé est de 30\%. Ainsi, le nombre minimum d'enfants de 0 à 59 mois à enquêter est estimé à 322.

\section{Matériel et méthodes d'étude}

Le recueil des données anthropométriques a été réalisé selon les recommandations de l'OMS (OMS, 2006). Une balance suspendue et un pèse-personne de type SECA avec une précision de $100 \mathrm{~g}$ ont été utilisés pour la prise du poids. La taille a été prise à l'aide d'une toise de précision 1 $\mathrm{mm}$ : pour les enfants de moins de 2 ans, la toise a été mise en position horizontale sur une surface plane et parallèle au sol. Pour les enfants de plus de 2 ans, la mesure a été effectuée en position debout avec une toise verticale. L'âge de l'enfant a été déterminé à partir de la date de naissance inscrite dans son carnet de vaccination, ou donnée par les parents. Les questionnaires à l'endroit des mères ou accompagnantes sont portés sur les caractéristiques socio-sanitaires de l'enfant, socio-économiques des parents, les pratiques alimentaires actuelles et antérieures de la mère, la consommation du plat familial et la diversification alimentaire de l'enfant.

\section{Classification des enfants}

Les indices anthropométriques calculés ont été : le Poids-pour-Age (P/A), la Taillepour-Age (T/A), le Poids-pour-Taille (P/T). La classification des sujets a été faite de la façon suivante: P/A : cet indice a permis de déterminer le pourcentage d'enfants ayant un poids insuffisant (P/A au-dessous de - $2 \mathrm{SD}$ ) et un poids gravement insuffisant (P/A au dessous de $-3 \mathrm{SD}$ ). T/A : cet indice a permis de déterminer le pourcentage d'enfants atteints de retard de croissance (T/A audessous de -2 SD) et de retard important de croissance (T/A au dessous de -3 SD). P/T : cet indice a permis de déterminer le pourcentage d'enfants émaciés (P/T audessous de -2 SD), gravement émaciés (P/T au dessous de $-3 \mathrm{SD}$ ), ayant un risque possible de surpoids (P/T au-dessus de $+1 \mathrm{SD})$, ayant un surpoids (P/T au-dessus de $+2 \mathrm{SD})$ et obèses (P/T au-dessus de +3 SD). (OMS, 2008).

\section{Saisie des données}

Toutes les données anthropométriques ont été saisies dans un dossier de surveillance nutritionnelle, créé à l'aide du logiciel WHO Anthro® version 3.2.2, 2011. Ce logiciel établit la valeur en percentile et en z-score de chaque paramètre, pour un groupe étudié. Il donne sous forme de courbe les répartitions pour chaque indice anthropométrique de la population d'étude par rapport aux standards de l'OMS (OMS, 2011).

\section{Traitement et analyse statistique des données}

Le traitement des données a été fait à

l'aide des logiciels Excel, WHO Anthro® 
version 3.2.2, 2011 et SPSS. Le test de khi2 en analyse uni variée a été utilisé pour rechercher une association entre les différents facteurs et l'état nutritionnel des enfants. Plus la valeur de chi2 est grande et moins le test est significatif $(\mathrm{p}<0,05)$.

\section{Considérations éthiques}

L'enquête a été réalisée suite à l'autorisation des autorités compétentes selon la note de service $\mathrm{N}^{\circ} 087$ de janvier 2016 de la Zone Sanitaire Porto-Novo/Aguégués/SèmèPodji. L'enquête n'a été menée qu'après obtention du consentement en préambule de la fiche d'enquête. La confidentialité des données a été garantie, l'identité de l'enfant n'a pas été dévoilée, chaque enfant a été représenté par un numéro.

\section{RESULTATS}

Sur un total de 350 enfants de moins de 5 ans à prédominance masculine (sex-ratio 1,12 ) reçus pour la vaccination, $83,1 \%$ des enfants de moins d'un (01) an ont été surreprésentés. Selon l'indice P/T, 7,4\% des enfants étaient émaciés dont $4,3 \%$ gravement émaciés, $21,1 \%$ présentaient un risque de surpoids, $13,1 \%$ étaient en surpoids et $11,7 \%$ d'obésité. La surcharge pondérale touchait $65 \%$ des enfants âgés de 0 à 5 mois. Par ailleurs, $30,3 \%$ des enfants accusaient un retard de croissance dont $16,9 \%$ de cas sévères. $12,3 \%$ des enfants enquêtés présentaient d'une insuffisance pondérale dont $4 \%$ de poids gravement insuffisant (Figure 1). Les courbes de distribution de la population enquêtée comparativement aux courbes de référence respectives sont décalées vers la gauche pour les courbes P/A et T/A (Figure 2, Figure 3) et vers la droite pour la courbe $\mathrm{P} / \mathrm{T}$ (Figure 4). Les différents tests de khideux ont montré de l'existence d'une relation significative entre les pratiques de sevrage et émaciation $(p=0,000)$ et insuffisance pondérale $(\mathrm{p}=0,002)$. Par contre, pas de relation significative avec le retard de croissance $(\mathrm{p}=0,381)$

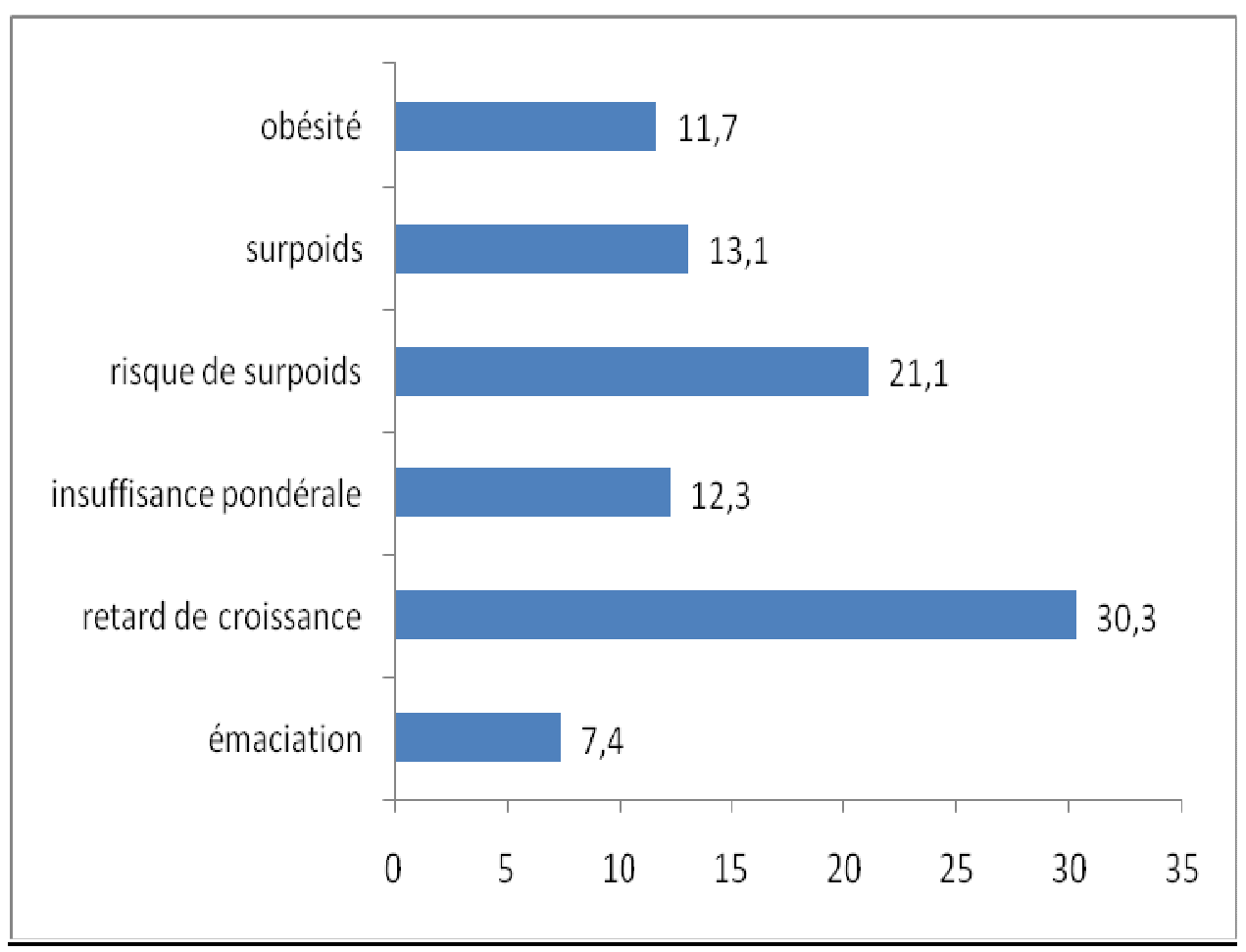

Figure 1 : Prévalences des différentes formes de malnutrition au sein de la population enquêtée. 


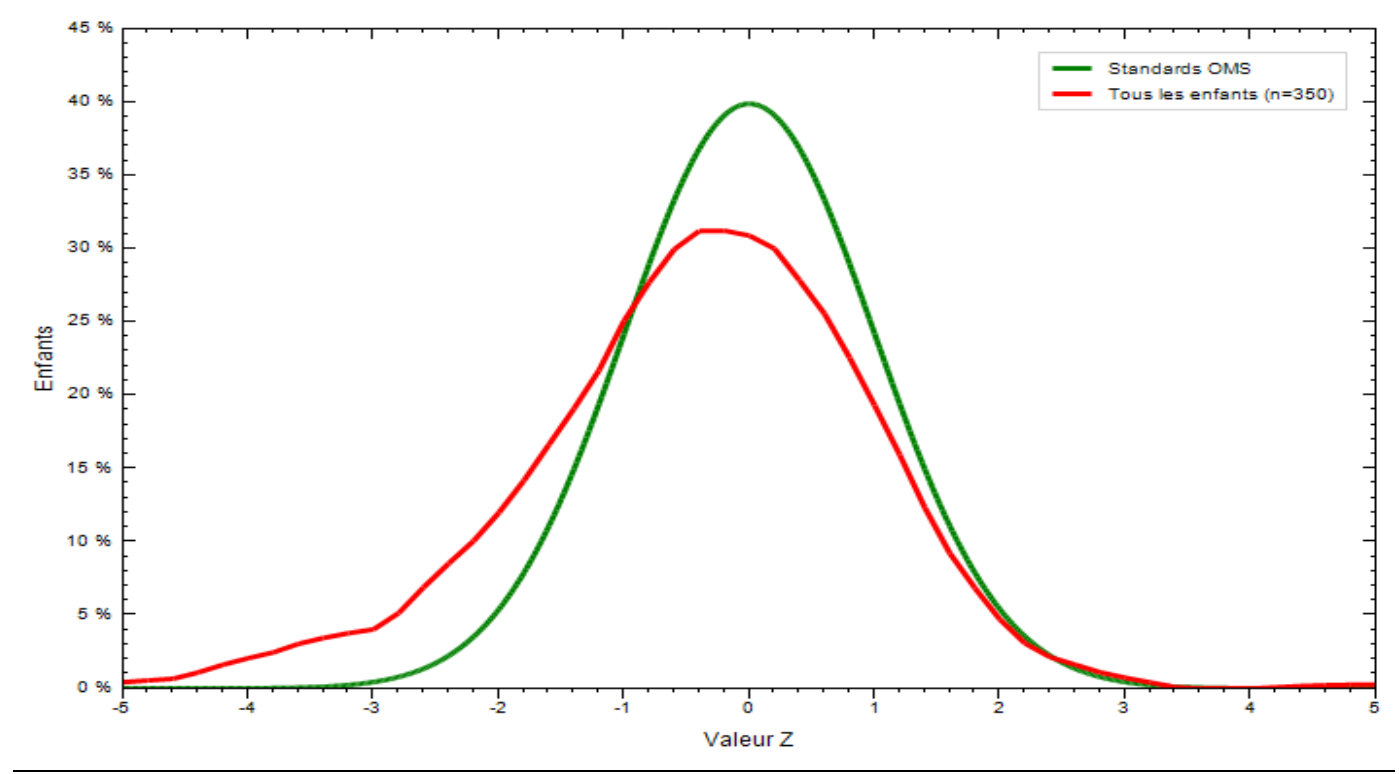

Figure 2: Distribution de l'indice Poids/Age en z-score par rapport aux standards OMS.

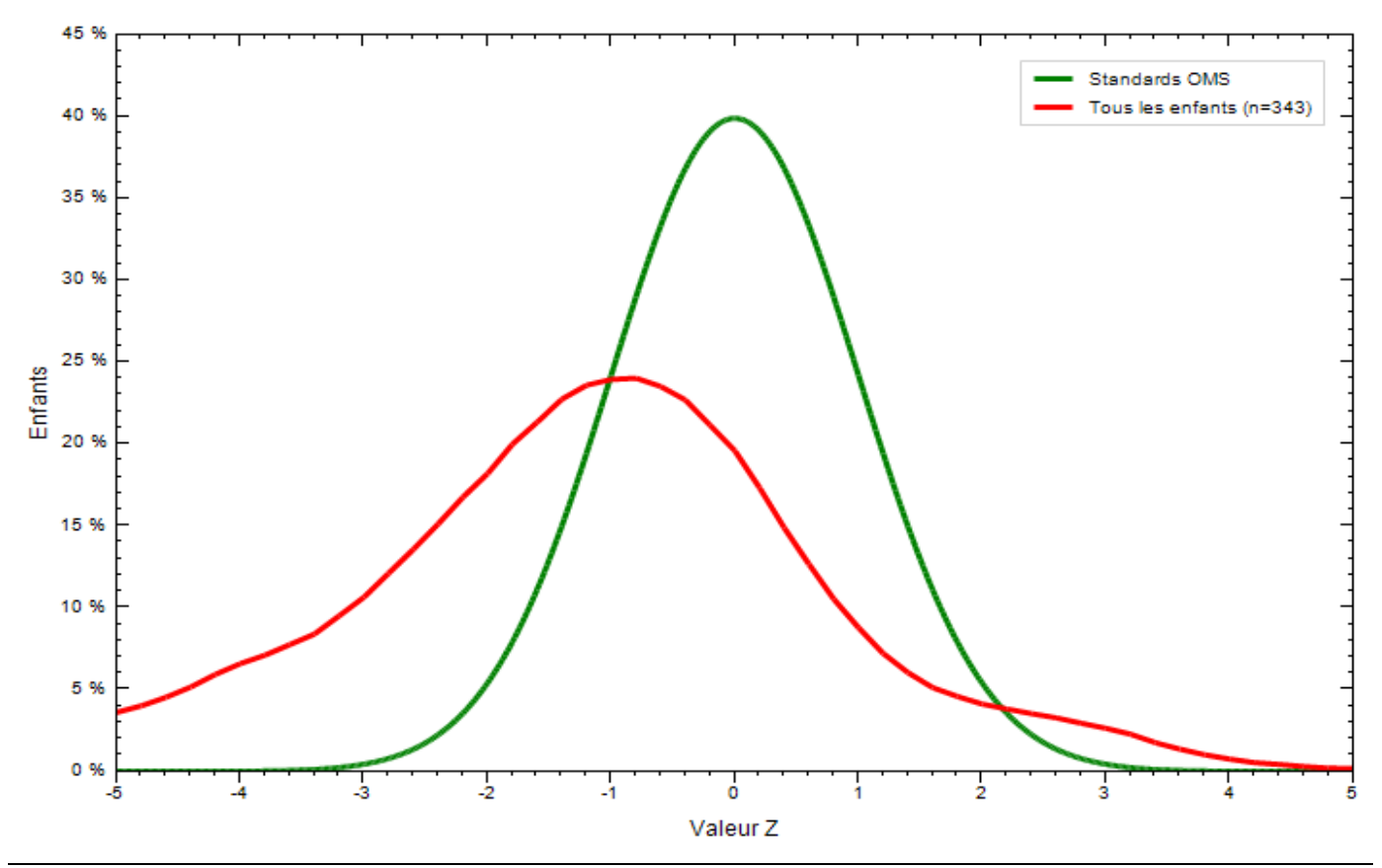

Figure 3: Distribution de l'indice Taille/Age en z-score par rapport aux standards OMS. 


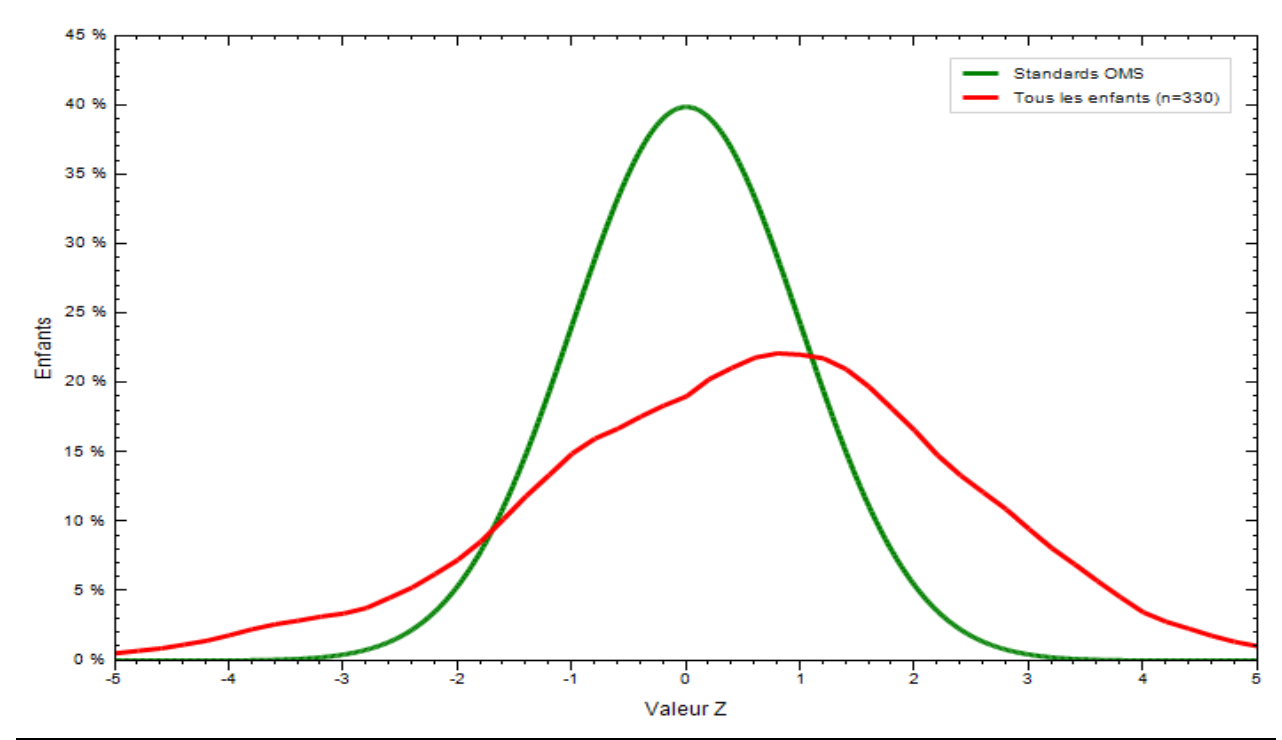

Figure 4: Distribution de l'indice Poids/Taille en z-score par rapport aux standards OMS.

\section{DISCUSSION}

Cette étude a révélé que $7,4 \%$ des enfants étaient émaciés, $30,3 \%$ présentaient un retard de croissance et $12,3 \%$ avaient une insuffisance pondérale. En ce qui concerne l'insuffisance pondérale et l'émaciation, ces résultats sont semblables à ceux de Gnimi et al (2013) en République du Congo dans leur étude faite sur l'évaluation de l'état nutritionnel des enfants de 0 à 59 mois (soit respectivement $8,4 \%$ et $10,8 \%$ ). Par contre, la prévalence de la malnutrition chronique obtenue est supérieure à celle du Congo $(20,3 \%)$. Les prévalences de retard de croissance et celle de l'émaciation de cette étude sont proches de celles obtenues dans le département de l'Ouémé par l'Analyse Globale de la Vulnérabilité et de la Sécurité Alimentaire (AGVSA, 2014) qui sont respectivement de $29,5 \%$ et $6,1 \%$. Par contre, la prévalence de l'insuffisance pondérale obtenue est inférieure à celle du département qui était de 15,7\%. Par ailleurs, les prévalences obtenues pour les différentes formes de malnutrition (émaciation, retard de croissance et insuffisance pondérale) sont supérieures à celles de l'étude menée à Lomé en 2014 qui sont respectivement de 3,7\%,
$12,5 \%$ et $7,5 \%$ (Yessoufou et al., 2015). Cette différence pourrait s'expliquer par le niveau socio-économique des populations de chaque étude et à des stratégies de lutte contre la malnutrition dans chaque pays. Aussi n'est-il pas aisé de constater que $45,9 \%$ des enfants présentaient une surcharge pondérale. Ce qui pourrait être justifié par le statut socioéconomique des populations de Porto-Novo avec plus de $2 / 3$ des ménages qui sont en sécurité alimentaire (AGVSA, 2014). Par ailleurs, $68 \%$ des mères des enfants menaient des activités génératrices de revenus leur permettant une prise en charge alimentaire adéquate de leur progéniture. Par conséquent, ayant accès facilement à la nourriture, elles ont tendance à exagérer dans l'alimentation complémentaire de leur enfant. Aussi, 65\% des enfants enquêtés âgés de 0 à 5 mois avaient une surcharge pondérale. Ces résultats sont comparables à ceux obtenus par Yessoufou et al. (2015) qui ont réalisé leur étude sur les enfants reçus à l'Unité de Vaccination du Centre Hospitalier Régional Lomé-Commune au Togo. Cette situation s'expliquerait par le fait que les mères n'auraient pas respecté le principe de l'allaitement maternel exclusif durant les six 
premiers mois de vie, recommandé par l'UNICEF/OMS. L'allaitement maternel est une source sûre et hygiénique d'alimentation suffisante d'énergie, d'éléments nutritifs et de liquides. Les mères ayant certainement de revenus confortables, alimentent leurs enfants de lait de vache en poudre et/ou du lait maternel, ce qui pourrait être à la base de la surcharge pondérale (excès de poids, obésité) observée chez les enfants de cette tranche d'âge au sein de la population d'étude. En effet, le lait de vache contient 3 fois plus de protéines que le lait maternel $(3,2 \mathrm{~g} / 100 \mathrm{ml}$ contre $1 \mathrm{~g} / 100 \mathrm{ml}$ ) (Nestlé/Nutrition, 2005). Les apports élevés en protéines chez les nourrissons sont responsables d'une forte activité métabolique chez l'enfantce qui pourrait conduire à une surcharge pondérale précoce (Verduci et al., 2014). La comparaison des courbes P/A et T/A avec les courbes de référence respectives, montre que ces courbes ont été décalées vers la gauche par rapport aux courbes de référence. Ce qui exprime un état nutritionnel insuffisant par rapport à celui de la population de référence donc une situation de sous-alimentation. Par contre, la courbe P/T est décalée vers la droite par rapport à sa courbe de référence traduisant un état nutritionnel excessif par rapport à la population de référence ; évocateur d'une situation de surnutrition. Ce fut le cas dans l'étude réalisée au CHR de Lomé-Commune par Yessoufou et al. (2015). Il ressort de cette analyse que la population d'étude est confrontée aux deux types de malnutrition (sous-nutrition et surnutrition). Une association statistiquement significative a été observée entre l'émaciation, l'insuffisance pondérale et les pratiques de sevrage ce qui permet de déduire que la cause de cette malnutrition était la mauvaise technique de sevrage comme l'ont souligné plusieurs auteurs (Barthélémy, 2002 ; Koné, 2008 ; Yessoufou et al., 2016).

\section{Conclusion \\ Cette étude a mis en évidence \\ l'existence d'une situation de double}

« charge » de la malnutrition : dénutrition et surnutrition. La surnutrition concerne de plus en plus des enfants des pays en développement, d'où un nouveau problème de santé publique dans l'avenir. La mauvaise pratique de sevrage serait l'un des déterminants associés à la malnutrition des enfants de moins de 5 ans dans cette zone. Par conséquent, un dispositif de surveillance régulière de croissance doit être mis en place en vue d'une prise en charge adéquate de ce fléau.

\section{CONFLIT D'INTERETS}

Les auteurs déclarent qu'il n'y a aucun conflit d'intérêt.

\section{CONTRIBUTIONS DES AUTEURS}

AAA et LE ont collecté et traité les données de l'enquête. AGY et AKY ont analysé, interprété les résultats et rédigé l'article. AS a supervisé l'enquête.

\section{REMERCIEMENTS}

Les auteurs remercient le Ministère de la Santé du Bénin à travers le Médecin Coordonnateur de la Zone Sanitaire de PortoNovo/Aguégués/Sèmè-Podji, le personnel du Centre de Santé de Hounsouko et celui du Laboratoire de Biomembranes et signalisation cellulaire de la FAST/Université d'AbomeyCalavi pour leur soutien indéfectible.

\section{REFERENCES}

AGVSA (Analyse Globale de la Vulnérabilité et de la Sécurité Alimentaire) 2014. Analyse globale de la vulnérabilité, de la sécurité alimentaire et de la nutrition. République du Bénin. p.142.

Barthélémie L. 2002. Approche d'identification des éléments concourant à l'apparition d'une malnutrition protéino-énergétique en Guyane française. Thèse de médecine, Université de Nantes, p. 162.

Gnimi Ch, Kibangou HS. 2013. Rapport anthropométrique. Projet PAM. République du Congo. p. 28. 
Kone M. 2008. Stratégies des ménages et malnutrition infantile dans la région de Madarounfa. Afrique Contemporaire,

1(225) : 161-197. DOI : http://dx.doi./10.3917/afco.225.0161

Nestle/Nutrition, 2005. Le lait maternel : en quoi sa composition est- elle si différente de celle du lait de vache. Mise à jour le 6 mai 2015; https://www.nutripo.Nestlé.fr consulté le 14 juin 2016 à $18 \mathrm{~h} 30$.

OMS. 2006. Normes OMS de croissance de l'enfant Longueur/taille pour l'âge, poids pour l'âge, poids pour la longueur, poids pour la taille et indice de Quételet pour l'âge. Méthodes et élaboration. Département Nutrition, santé et développement. $5 \mathrm{p}$.

OMS 2008. Cours de Formation sur l'Evaluation de la Croissance de l'Enfant. Genève : OMS. Kit documentaire de 13 modules.

OMS 2010. Le guide d'utilisation des nouvelles courbes de croissance de l'OMS à l'intention du professionnel de la santé. Paediatr Child Health. 15(2): 91-98.

OMS 2011. Succès des nouvelles normes OMS de croissance de l'enfant. Bulletin de l'OMS, 89(4): 250-251. DOI : http://dx.doi.org./10.2471/BLT.11.040411

Perlemuter G. 2002. Bilan nutritionnel. In Endocrinologie-Diabétologie-Nutrition. Paris: Edition Med-Line; p. 225.

Rice AL, Saccl L, Hyder A, Black RE. 2004. La malnutrition en tant que cause sousjacente des décès par les maladies infectieuses chez les enfants dans les pays en développement. Bull World Health Organ, 78(10): 1207-1221. DOI: http://dx .doi.org/10.1590/S004296862000001000007

Sakineh NS, Fariba B, Parvin A. 2014. Malnutrition, overweight, and obesity among Urbain and Rural children in North of West Azerbaijan, Iran. Journal of Obesity, Vol. 2014, Article ID 541213, 5 pages, DOI: http:// dx.doi.org/10.115/2014/541213

UNICEF (Fonds des Nations Unies pour l'Enfance) 2009. Suivre les progrès dans le domaine de la nutrition de la mère : New York. p.76.

Verduci E, Mariani B, Lassandro C, Redionigi A. 2014. Protein intake and nutritional programming: Metabolic consequences. Italian Journal of Pediatrics, 40(1): A 49. DOI: $\quad$ http://dx.doi.org./10.1186/18247288-40 S1-A49

Yessoufou. AG, Yessoufou KA, Gbaguidi B, Sezan A, Agbere A-RD. 2015. Evaluation anthropométrique de l'état nutritionnel des enfants de 0 à 59 mois reçus à l'Unité de vaccination du CHR Lomé-Commune (Togo). J. Rech. Sci. Univ. Lomé (TOGO), 17(1) : 21-32.

Yessoufou AG, Vinou AY, Gbaguidi B, Affognon JK, Baba-Moussa LS, Sezan A. 2016. Diversity in food and nutritional status in children from 6 to 59 months in the municipality of Abomey-Calavi Southeast of Benin (West Africa). Int J Ad Rech, 4(2): 594-600. DOI: http:/dx.doi.org./10.21474/IJAR01 